


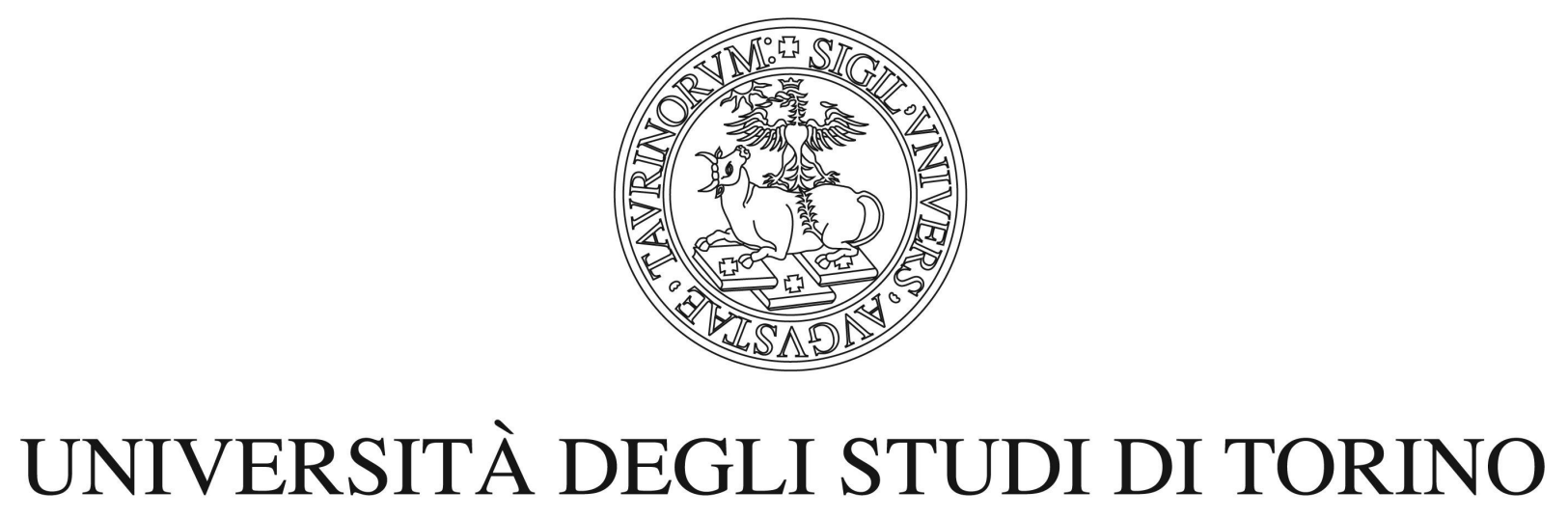

This is an author version of the contribution published on:

Questa è la versione dell'autore dell'opera:

"Presentation of a research project addressed to the realisation of a diamond-based cellular biosensing device"

Proceedings of SPIE 8207 (2012)

DOI: $10.1117 / 12.916749$

The definitive version is available at:

http://proceedings.spiedigitallibrary.org/proceeding.aspx?articleid $=1344256$ 


\title{
PRESENTATION OF A RESEARCH PROJECT ADDRESSED TO THE REALISATION OF A DIAMOND-BASED CELLULAR BIOSENSING DEVICE
}

\author{
Luca Boarino $^{\mathrm{a}}$, Valentina Carabelli ${ }^{\mathrm{c}, \mathrm{e}}$, Emilio Carbone ${ }^{\mathrm{c}, \mathrm{e}}$, Marco Genovese ${ }^{\mathrm{a}}$, Paolo Olivero ${ }^{\mathrm{b}, \mathrm{d}, \mathrm{e}}$, \\ Sara Gosso ${ }^{\mathrm{c}, \mathrm{e}}$, Alberto Pasquarellif ${ }^{\mathrm{f}}$, Federico Picollo ${ }^{\mathrm{d}, \mathrm{e}}$, Paolo Traina ${ }^{\mathrm{a}}$ \\ a INRIM, 91 Strada delle Cacce, Torino, I 10135 \\ ${ }^{\mathrm{b}}$ CNISM, 30 Corso Raffaello, Torino, I 10125 \\ ${ }^{\mathrm{c}}$ Department of Neuroscience, Univ. of Torino, 30 Corso Raffaello, Torino, I 10125 \\ ${ }^{\mathrm{d}}$ Department of Experimental Physics, Univ. of Torino, 1 Via P. Giuria, Torino, I 10125 \\ ${ }^{\mathrm{e}}$ NIS Centre of Excellence, Univ. of Torino, 7 Via P. Giuria, Torino, I 10125 \\ ${ }^{\mathrm{f}}$ Institute of Electron Devices and Circuits, Ulm University, 89081 Ulm, Germany
}

\begin{abstract}
In this proceedings we will present a research project financed by Piedmont regional government (Italy) and finalised to the realization and commercialization of functional devices for cellular bio-sensing based on diamond. Partners of the project are: Crisel Instruments, Torino University, Torino Polytechnic, INRIM, Politronica, Bionica Tech, Ulm Unniversity

Here the main features of the final devices will be briefly summarized.

We envisage an active diamond-based cellular substrate that can simultaneously stimulate and detect a variety of signals (chemical, optical, electrical) to and from neuroendocrine cells, in a fully biocompatible environment for the cellular system under test. Such a device can be realized by fully exploiting the peculiar properties of diamond: optical transparency, biocompatibility, chemical inertness, accessibility to a conductive graphite-like phase; properties that will be further explored and tested during the project.
\end{abstract}

Keywords: cellular bio-sensors, nanocrystalline and monocrystalline diamond surfaces, ion beam lithography.

\section{INTRODUCTION}

The diamond biosensor that we are considering will be based on the realization of the following basic components:

- nanocrystalline boron-doped diamond detecting electrodes, patterned with different geometries to perform amperometric and potentiometric measurements from excitable cells;

- buried conductive paths (fabricated in single-crystal diamond by $\mathrm{MeV}$ ion implantation in defined paths) will contact the neuronal cells surface and enable their electrical stimulation, as well as the electrical recording of their action potential signal via voltammetric measurements, or the quantal release of biomolecules by means of amperometric recordings.

This innovative diamond-based biosensor will present several advantages with respect to the current cellular sensing devices: namely, the possibility of integrating a series of different interfacing structures (microfluidic channels in standard polymeric materials, conductive pathways) in a single device which is fully biocompatible and optically transparent, thus allowing to simultaneously 
record and stimulate a range of different signals (chemical, electrical and optical) while being able to monitor the biological system through a standard microscope with back illumination of the active substrate.

\section{THE MICRODIBI PROJECT}

More in detail the MiCroDiBi project is aimed at the development of cellular bio-sensors.

The micro-fabrication and micro-functionalization techniques most suitable for diamond microdevices production will be developed on the basis of recent results obtained by the UniTo group.

Such techniques can be briefly described as follows:

1) fabrication on mono-crystalline diamond via ion lithography: By means of ion beams with energy of the order of $\mathrm{MeV}$ (available to UniTO unit (NIS-DFS) through the access to major ion microbeam facilities) it is possible to structure the material on the micrometric scale and realize sub surface conductive channels by localized conversion at a graphitic phase; suitable lithographic methods allow the deep structuring of the channels as it has been already proven by members of UniTO group (NIS-DFS) in several publications [1-4].

2) development of surface functionalization techniques: oxidation and hydrogenation techniques will be optimized on the basis, respectively, of exposition treatments to ozone and hydrogen plasma, which have already been successfully developed at UniTo group; by means of these treatments it will be possible to functionalize the properties of electro-conduction and cellular adhesion of the diamond surface. Furthermore, FIB focused ion beams micro-structuring at INRIM will allow the morphologic functionalization of the material to optimize the cells selective adhesion to the surface.

3) CVD growth of boron-doped nano-crystalline diamond and insulation of bio-sensitive microareas with photoresist material.

This work will be performed by UniTo group (NIS) in collaboration with Ulm University. A nanocrystalline boron doped diamond microchip will be built capable of detecting electrical and electrochemical signals (adrenaline, dopamine and serotonin levels) from neuroendocrine cells. The new multi-electrode $(\mathrm{NCD}-\mathrm{MEA})$ will have $3 \times 3$ detecting areas that will occupy a total square area of about $300 \mu \mathrm{m}^{2}$, corresponding to the surface of $20 \mu \mathrm{m}$ diameter cell. In the subsequent models, each detecting electrode will cover an area $300 \mu \mathrm{m}^{2}$, separated each other by $200-300 \mu \mathrm{m}$. This configuration is suitable for detecting events from cell populations.

Therefore, while the scientific scope of the project is the development of an innovative platform for the fabrication of diamond, the final goal is strongly oriented towards the application of innovative devices, for which significant scientific and technological outcomes are expected. 


\section{STATE OF THE ART}

The present project is prompted from the fact that "Lab-on-chip" devices are highly demanded in modern biotechnology to cultivate for long terms living cells while inducing and revealing a broad range of electrical (nerve excitations, synaptic transmissions), chemical (absorption/release of molecules and ions) and optical (photo-excitation, luminescence) bio-signals. Interfacing living tissues and single cells with miniaturized devices easily interfaced with conventional instrumentation (microscopes, electrometers, fluidic devices) will certainly find many applications in both fundamental research and industry, ranging from pharmacology to cell diagnostic.

For decades diamond have attracted scientific interest due to its unique properties such as high mechanical hardness and thermal conductivity, chemical inertness and stability, biocompatibility, high optical transparency.

More recently, after the discovery of the possibilities offered by its surface chemical termination, the study of the diamond surface functionalization as an interface to biological systems has attracted growing interest and quickly developed into a vital research field at the crossover between material science, chemistry and biophysics.

In recent years high quality artificial diamonds have become available at ever decreasing prices. As an example, $1 \mathrm{~cm} \times 1 \mathrm{~cm} \times 6 \mathrm{~mm}$ polycrystalline CVD diamond samples for electrochemistry application are commercially available in large quantities at the price of about $30 €$ per piece, while $2.6 \mathrm{~mm} \times 2.6 \mathrm{~mm} \times 0.5 \mathrm{~mm}$ single-crystal samples produced with HPHT technique are available at about $38 €$ per piece.

These significant advancements in the material synthesis paved the way to the use of artificial diamond as a viable alternative to standard materials for specific bio-sensing applications.

A diamond-based bio-device would effectively address many open issues in the existing bio-sensing research, which are not met by conventional bio-materials (silicon, metals and metals oxides, polymers): robustness and reproducibility in performance over repeated bio-sensing cycles, biocompatibility and long term stability for in vivo measurements, surface selectivity to different cellular and bimolecular bonding, high transparency for optical interfacing, broad electrochemical window.

The primary scope of the proposed device consists in the electrical stimulation and detection of action potentials (APs) with graphitic or boron-doped microelectrodes embedded in a fully biocompatible and transparent diamond substrate. APs are defined as fast (around 10-100 ms) membrane depolarizations (from $-60 \mathrm{mV}$ to $+50 \mathrm{mV}$ ) due to the entering into the cell of sodium and calcium ions, and represent one of the most important physiological process that govern the synaptic neurotransmitter release and intercellular communication. Different frequencies and patterns of action potentials generation (firing of a rapid series of several action potentials called bursts, or action potentials generated at a constant frequency) can differently modulate the neurotransmitter release and can be investigated with conventional multielectrode array devices (MEAs), with significant advantages in the case of a diamond substrate. For instance, the possibility of directly creating buried contacts is extremely useful for the proposed application, in which the bio-system of interest (typically an endocrine cell) is constantly kept in a suitable culturing liquid medium over all the measurement process. In standard MEAs used of AP measurements, the electrical insulation of the micro-contacts is realized with fabrication strategies involving masking steps with insulating materials, while in the proposed device the buried electrodes would be directly created in the active 
diamond substrate. Buried electrodes are required to emerge and get in contact with the sample surface at specific locations where the cellular AP sensing is designed to occur.

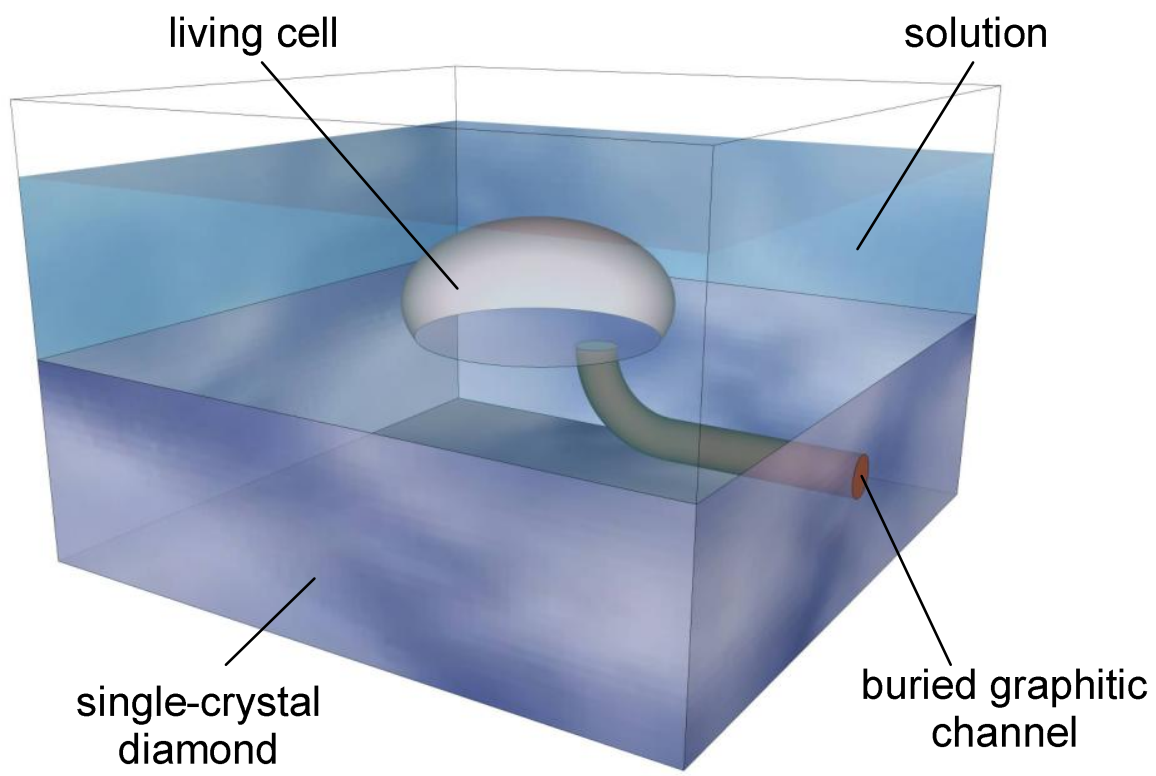

Figure 1 schematics of an cellular bio-sensor in diamond for bio-electrical sensing. The cell is electrically interfaced with a subsuperficial conductive microchannel

Impairment and malfunction of synaptic transmission is the cause of various mental disorders. As synaptic transmission is controlled by a multitude of events (ion channel modulation, intracellular $\mathrm{Ca}^{2+}$ concentration, membrane receptor activation, neurotransmitter release and re-uptake) the origin of most mental illness (depression, schizophrenia, Alzheimer's disease) is open to a variety of possible impaired molecular mechanisms that remain largely unknown. For these reasons, it is widely recognized that, in the contest of bio-sensing and neuroscience, the study of mechanisms that govern the cell excitability is considered of the utmost importance. A strategic open field is the ongoing search for new drugs designed for the treatment of the above mentioned mental disorders, therefore the possibility of implementing waveguides in diamond add a whole new dimension, which would enable the multi-parametric acquisition of the above-mentioned APs together with luminescence detection of specific ionic species (i.e. intracellular calcium) with ratiometric dyes. Additionally, the possibility of optically interfacing the cell would allow the stimulation of caged compounds for the controlled release of inert precursors of active molecules (ions, neurotransmitters, metabolites, etc.), which become available within the extra-cellular medium or in the citosol, by releasing active species at the site action upon photo-excitation.

\section{METHODOLOGY}

Realization of nanocrystalline diamond microelectrodes 
Biosensors made with nanocrystalline boron-doped diamond electrodes were realized as described by Carabelli et al., 2010. Starting from a single crystal double-polished 330 microm thick sapphire wafer, a Si-based nucleation layer was first deposited by PECVD (Plasma Enhanced Chemical Vapor Deposition). The formation of nanodiamond nuclei was driven by Bias Enhanced Nucleation (BEN) in a HFCVD (Hot Filament CVD) system. Onto this substrate a 200nm layer of intrinsic NCD was grown and as a final step, a quasi-metallic boron doped top layer of $350 \mathrm{~nm}$ thickness was deposited. Realization of the mask for patterning the microelectrode array was performed by optical lithography and then dry etching in an O2/Ar plasma down to the sapphire substrate. The electrochemically active areas of the microelectrode array were finally defined by patterning an SU8 passivation layer with four circular openings of $20 \mu \mathrm{m}$ in diameter onto the chip surface.

\section{Realization of graphitic micro-electrodes in single-crystal diamond}

Buried microelectrodescan be applied to create a single-cell biosensor (se fig.1) able to convert in electrical signal (extracellular AP) a biological signal resulting from the interaction between a specific neurotrophins injected at the single-cell scale and the corresponding membrane receptor on the cell.

Despite this great scientific and technological potential, and the above-described commercial availability of high quality synthetic diamonds, the systematic micro-fabrication of cell-sensing devices in single-crystal diamond has not been extensively explored. This is due to the fact that the same properties that make diamond so attractive (in particular: its hardness, chemical inertness, high electrical resistance) also make its nano-fabrication extremely challenging: we are filling this significant technological gap with new fabrication strategies.

In particular we are achieving this result through the development of a three-dimensional lithographic technique on single crystal diamond based on the integration of the deep ion beam lithography (DIBL), which is based on the employment of focused ion beams with energy of the order of $\mathrm{MeVs}$, with more conventional lithographic techniques: focused ion beam (FIB) milling with ions with energies of the order of $10 \mathrm{keV}$.

The fabrication process offers unique advantages due to the peculiar interaction of $\mathrm{MeV}$ ions with matter. The $\mathrm{MeV}$ ions, having a greater mass and energy with respect to $\mathrm{keV}$ electrons, penetrate to a greater depth, while still maintaining a near straight trajectory; the interaction with the material atoms is essentially localized at end of range and induces a displacement from their reticular location that causes a partial or complete amorphization of the latter. This allows the realization of buried microelectrodes whose dimensions are defined by the area scanned by the focused beam and whose depth is determined by the range and longitudinal straggling of the employed ions.

Fig. 3a shows a schematic representation of the definition of sub-superficial micro-electrodes in diamond by means of $\mathrm{MeV}$ ion beam implantation through variable-thickness masks, to make the channels emerge at the sample surface in correspondence of specific locations. Fig. 3b shows typical IV curves from micro-channels implanted with $\mathrm{He}$ and $\mathrm{C}$ ions at increasing fluences [p3]. 

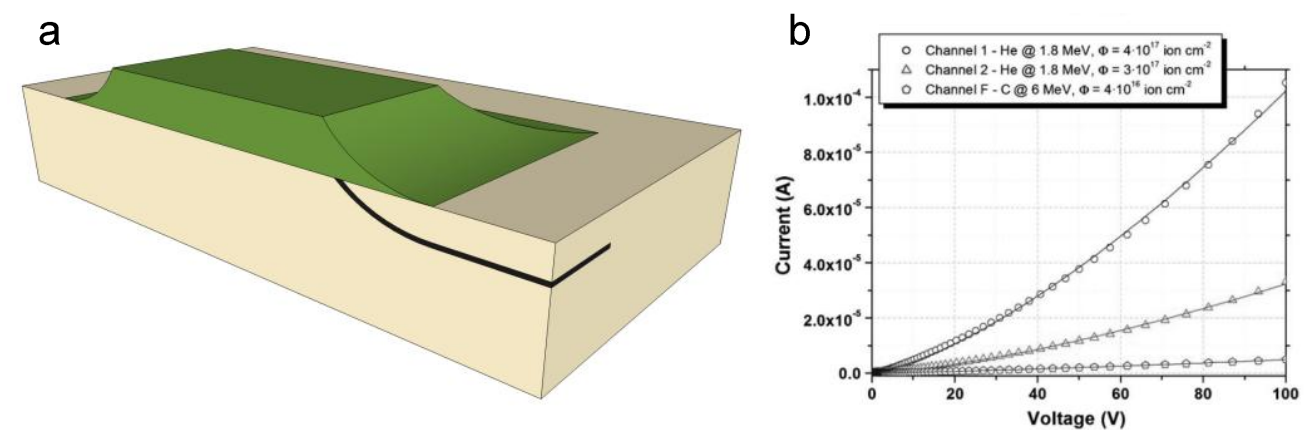

Figure 3: a) schematic representation of the definition of sub-superficial diamond microelectrodes by means of $\mathrm{MeV}$ ion beam implantation through variable-thickness masks; b) IV curves from micro-channels implanted with $\mathrm{He}$ and $\mathrm{C}$ ions at increasing fluences [p3].

\section{PRELIMINARY RESULTS}

Some demonstrative applications of the cellular biosensing systems have been achieved with prototypical devices (both based on nanocrystalline and single-crystal diamond) applied to chromaffin cells.

Bio-sensors in nanocrystalline diamond A first test concerned the detection of quantal release events of catecholamine. Exocytosis is a $\mathrm{Ca}^{2+}-$ dependent process at the basis of synaptic transmission occurring through quantal release of neurotransmitter molecules into the synaptic cleft. Single secretory events of oxidizable molecules can be conventionally detected by means of carbon fiber microelectrodes (CFEs), with submillisecond time resolution but limited to single cells. These preliminary results demonstrate the potentiality of a planar nanocrystalline diamond microelectrode array for recording quantal secretory events of oxidizable neurotransmitters from living cells. 


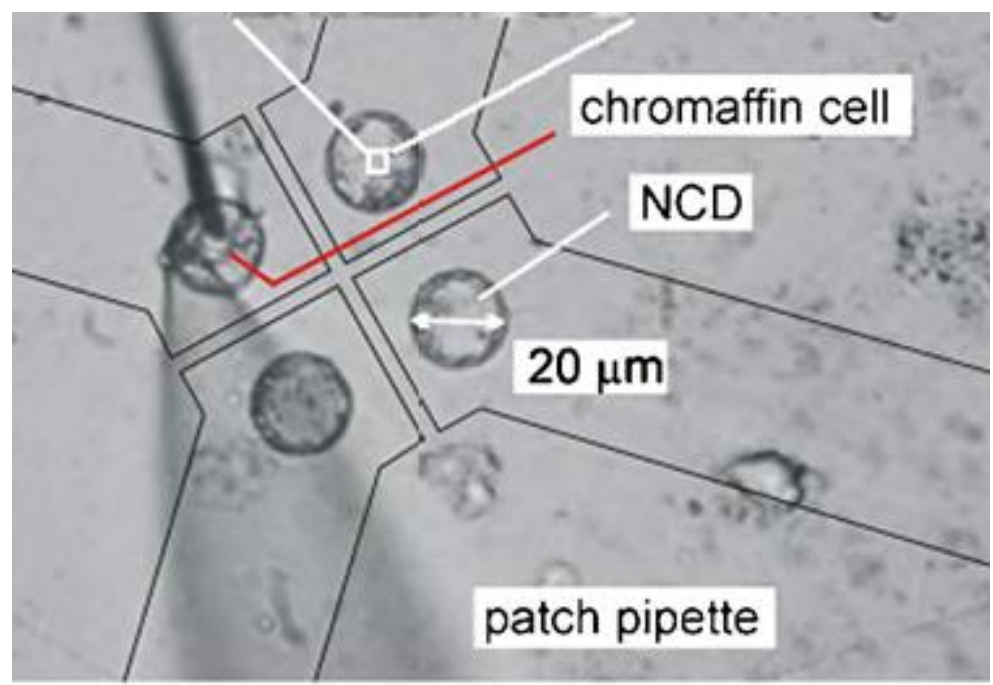

Figure 4: Light-microscope image of planar boron-doped NCD array with four detecting microelectrodes. A mouse chromaffin cell has been placed onto one microelectrode using a glass patch-pipette. A carbon fiber microelectrode was placed close to the cell membrane for the simultaneous detection of catecholamine secretion (adapted from [6]).

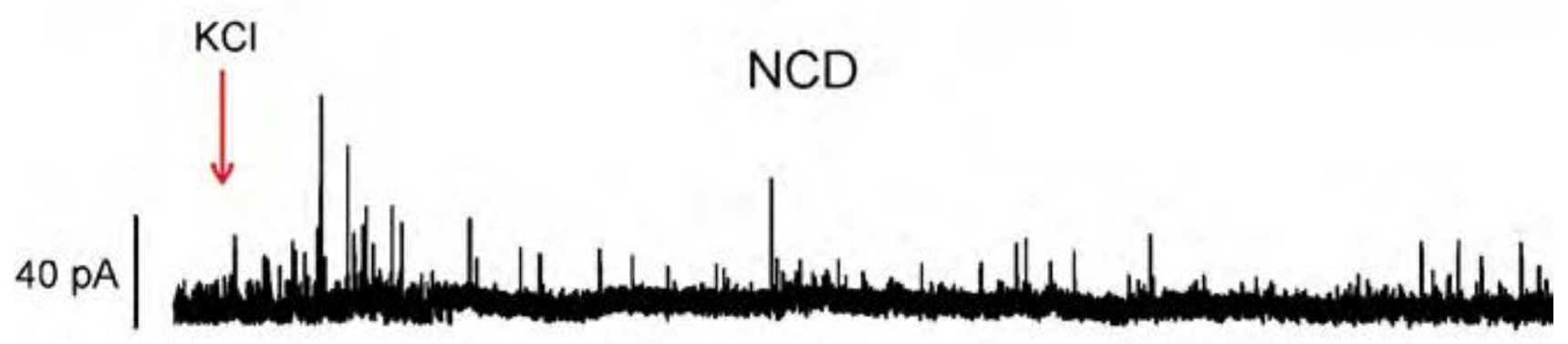

Figure 5.Quantal secretory events from mouse chromaffin cells detected the planar boron-doped NCD device. Mouse chromaffin cells were stimulated by external $\mathrm{KCl}(30 \mathrm{mM})$ at the time indicated by the arrows. 

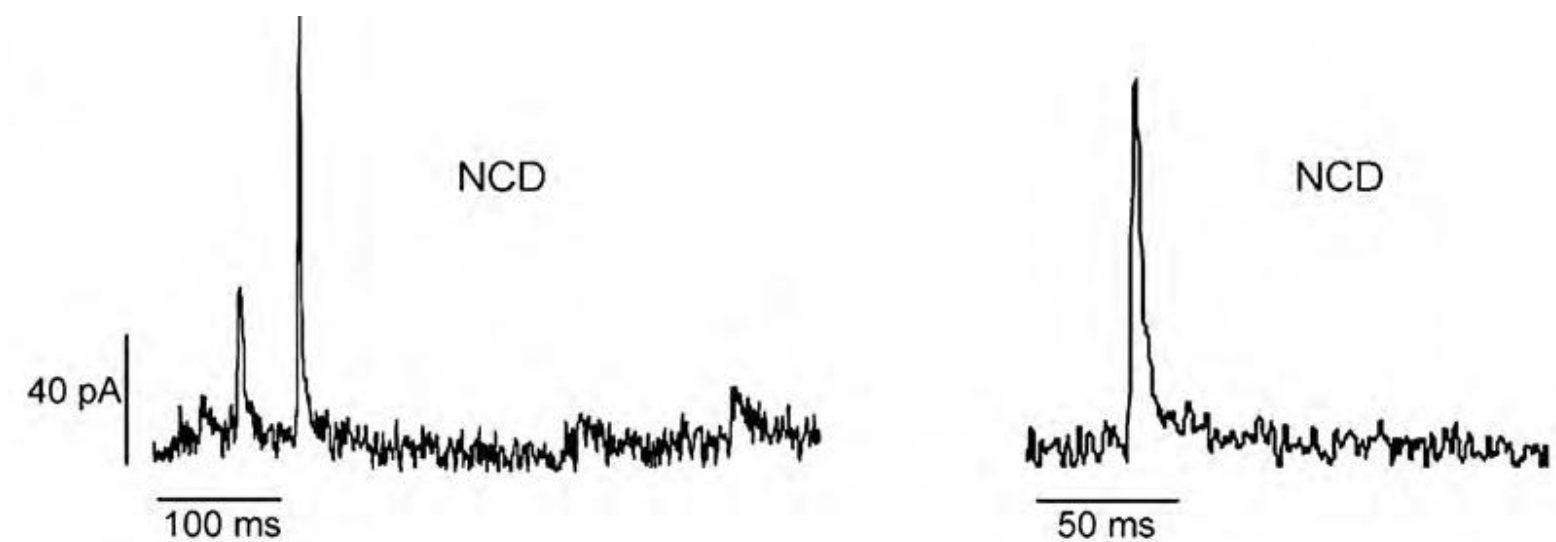

NCD

$50 \mathrm{~ms}$
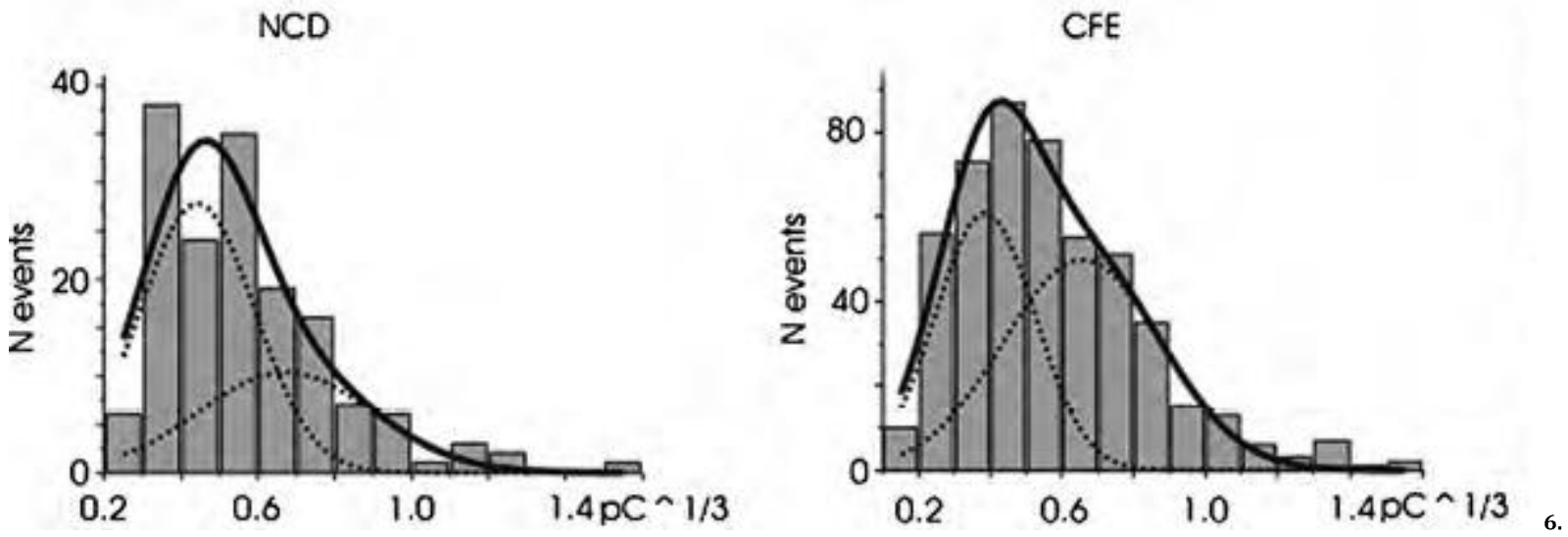

Figure 6. Comparison of amperometric spikes recorded using the conventional carbon-fibre microelectrode (CFE) and the NCD-based device. (A) Representative spikes obtained by the NCD microelectrode at two different time scales. Distribution of the cubic root of the vesicular charge (Q1/3) from amperometric spikes detected with NCD chips and CFEs. Data were fitted with a sum of two Gaussian functions (adapted from [6]).

Bio-sensors in single-crystal diamond

The above-mentioned DIBL fabrication techniques have been implemented in synthetic diamond grown with high-pressure-high-temperature (HPHT), to fabricate a prototypical bio-sensing device with sub-superficial graphitic microchannels acting as sensing cellular microelectrodes. An image of the preliminary device setup is shown in Fig. 7.

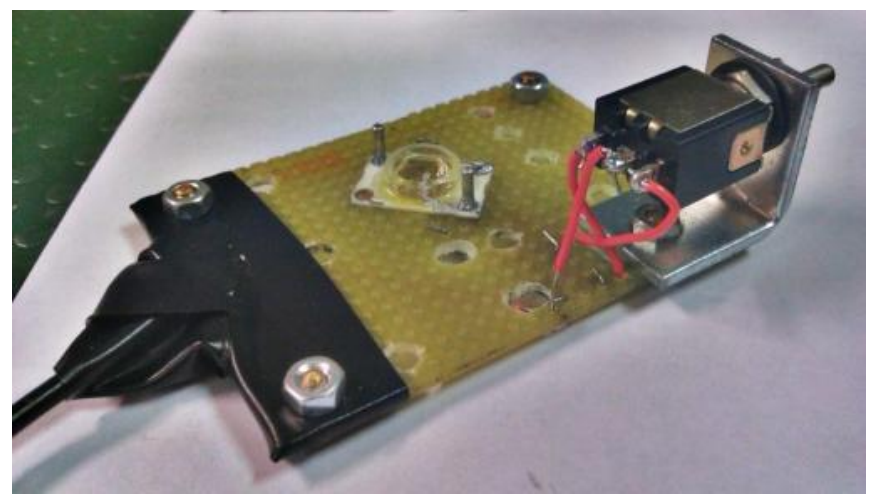

Figure 7: Prototype of a cellular biosensor based on artificial single-crystal diamond. 
Preliminary results from this device demonstrate the possibility of revealing the exocytotic activity of chromaffin cells with a sensitivity and temporal resolution comparable to that of the abovementioned nanocrystalline diamond-based device. The results are yet unpublished, but they have been presented at international conferences [5].

\section{ACKNOWLEDGEMENTS}

We acknowledge the contribution of Piedmont Region through MicroDiBi project of Innovation Pole Biomed and of Italian Minister of Research through FIRB project RBFR10UAUV. Part of this work will be performed at NanoFacility Piemonte, INRiM, a laboratory supported by Compagnia di San Paolo.

\section{REFERENCES}

[1] P. Olivero et al., "Direct fabrication of three-dimensional buried conductive channels in single crystal diamond with ion microbeam induced graphitization", Diamond Relat. Mater. 18, 870-876 (2009)

[2] P. Olivero et al., "Direct fabrication and IV characterization of sub-surface conductive channels in diamond with MeV ion implantation", Eur. Phys. J. B 75 (2), 127-132 (2010)

[3] F. Picollo et al., "Formation of buried conductive micro-channels in single crystal diamond with $\mathrm{MeV} \mathrm{C}$ and $\mathrm{He}$ implantation", Diamond Relat. Mater. 19, 466-469 (2010)

[4] P. Olivero et al., "Focused ion beam fabrication and IBIC characterization of a diamond detector with buried electrodes", Nucl. Instr. Meth. in Phys. Res. B 269, 2340-2344 (2011)

[5] E. Vittone et al., "A fully ion beam micromachined diamond biosensor designed for the detection of quantal catecholamine secretion from chromaffin cells", talk at MRS Fall Meeting (id: N6.2), 28 November-2 December 2011 , Boston (USA)

[6] V. Carabelli et al., "Nanocrystalline diamond microelectrode arrays fabricated on sapphire technology for high-time resolution of quantal catecholamine secretion from chromaffin cells", Biosensors and Bioelectronics 26, 9298 (2010) 